\title{
Avaliando o Uso da Ferramenta Scratch para Ensino de Programação através de Análise Quantitativa e Qualitativa
}

\author{
Hannah Belchior ${ }^{1}$, Bruno Bonifácio, Rallyson Ferreira ${ }^{1}$ \\ ${ }^{1}$ Instituto de Ciências Exatas e Tecnologia (ICET) \\ Universidade Federal do Amazonas (UFAM) \\ Caixa Postal 15.064 - 91.501-970 - Itacoatiara - AM - Brazil \\ \{hannahfarias21, brunnoboni, rallysonferreira@gmail.com\}
}

\begin{abstract}
A major challenge in computing and IT courses is teaching programming. Several approaches and tools have been proposed to facilitate and maximize the student's ability to solve problems using programming skills. However, students end up facing obstacles such as lack of knowledge in tools functionalities and the difficulty while interacting with the non-intuitive interfaces of applications. This paper presents an experimental study to evaluate the impact of communicability in the programming teaching-learning process. We use Scratch, a programming learning environment for beginner. The results show that these approaches can positively impact in the learning process. And also create a motivating environment for learning, providing a more intuitive learning experience.
\end{abstract}

Resumo. Um grande desafio em cursos de computação e TI é ensinar programação. Várias abordagens e ferramentas têm sido propostos para facilitar e maximizar a capacidade do aluno para resolver problemas usando as habilidades de programação. No entanto, os alunos acabam enfrentando obstáculos como a falta de conhecimento em funcionalidades de ferramentas e a dificuldade ao interagir com as interfaces não-intuitivas de aplicações. Este trabalho apresenta um estudo experimental para avaliar o impacto da comunicabilidade no processo de ensino-aprendizagem de programação. Nós usamos Scratch, um ambiente de aprendizagem de programação para iniciantes. Os resultados mostram que estas abordagens podem ter um impacto positivo no processo de aprendizagem. E também criar um ambiente motivador para o aprendizado, proporcionando uma experiência de aprendizagem mais intuitiva.

\section{Introdução}

A evolução tecnológica atual, tanto de hardware quanto de software, trouxe importantes mudanças no modo como seus usuários interagem e acessam as informações [Whang et al. 2009]. Esse cenário contribuiu para o surgimento de novos paradigmas, tanto relacionados ao acesso à informação quanto na forma de aprendizado dos usuários [Bonifácio et al. 2015].

No ambiente educacional, o uso de tecnologias de software para apoiar o ensino têm despertado grande interesse por fornecer a flexibilidade, melhorar a integração e por incentivar práticas de ensino e aprendizagem a partir de uma perspectiva pedagógica inovadora [Power e Shrestha 2010; Marçal et al. 2009]. O desafio com essa integração é criar novas formas de interação, com atividades pedagógicas mais colaborativas de 
ensino, tornando o processo de aprendizagem mais dinâmico, ágil e prazeroso [Ronnie et al. 2014].

Por essa razão, a adoção de softwares como ferramenta de apoio no ensino tem sido amplamente adotada em várias áreas [Fincher et al. 2010]. Na área de Tecnologia da Informação, essa abordagem é usada como mecanismo de ensino aos alunos visando maximizar a capacidade do aluno apresentar soluções para diversas classes de problemas, por meio de linguagens de programação. Como os métodos tradicionais de ensino não consideram as particularidades de aprendizado de cada aluno, torna-se importante utilizar uma abordagem que torne melhor sua experiência no aprendizado [Gomes e Mendes 2007].

Nesse contexto, várias ferramentas têm sido adotadas para o ensino de programação visando se adequar ao estilo, as necessidades e preferências de aprendizado de cada aluno. Grande parte das abordagens apresenta uma linguagem de programação visual e têm por objetivo apoiar os seus usuários no aprendizado de conceitos de programação de uma maneira mais divertida e estimulante [Cooper, Dann e Pausch 2000; Maloney et al. 2010]. No entanto, esse novo modelo educacional, acaba tendo como principais obstáculos a falta de conhecimento no uso das funcionalidades e a dificuldade de interação dos usuários associada às interfaces não-intuitivas das aplicações [Luna et al. 2010].

Por essa razão, a usabilidade tem adquirido papel fundamental, uma vez que aplicações fáceis ajudam a aumentar a satisfação de uso e pode impactar no aprendizado dos alunos [Nauman et al. 2007]. Assim, o presente artigo apresenta um relato de experiência sobre o uso do Scratch para ensino de programação, avaliando o impacto da usabilidade da ferramenta no aprendizado dos alunos. Os objetivos desse artigo consistem em: 1) apresentar como foi planejado e executado o processo de ensino, utilizando a ferramenta Scratch; 2) mostrar os resultados quantitativos e qualitativos que impactam na experiência dos alunos com a ferramenta; e 3) encorajar outras instituições e profissionais da educação na adoção do Scratch como alternativa para o processo de ensino e aprendizagem de programação.

As próximas seções deste artigo estão organizadas da seguinte forma: A Seção 2 descreve os conceitos e trabalhos relacionados, apresentando ainda as características do Scratch e o seu funcionamento. A Seção 3 apresenta a avaliação de usabilidade aplicada para melhorar a experiência de uso, e também discute os resultados obtidos de forma quantitativa e qualitativa. Por fim, a Seção 4 lista as conclusões e lições aprendidas.

\section{Background e Ambiente Introdutório de Programação Scratch}

O processo de aprendizagem na disciplina de algoritmos e lógica de programação tem sido um dos principais desafios encontrados nos cursos de graduação da área de Computação. Pesquisas revelam que tal disciplina apresenta taxas de reprovação e/ou desistência de até 75\% [Rocha et al. 2010; Pereira et al. 2012], além de gerar um alto índice de retenção, uma vez que os conhecimentos adquiridos nela influenciam diretamente no desempenho das demais disciplinas correlatas no decorrer do curso.

Com o intuito de minimizar essas dificuldades, várias abordagens têm sido propostas. O propósito com isso é a criação de novos ambientes introdutórios de ensino (Initial Learning Environments). Essa abordagem consiste da construção de ferramentas que apóiam o processo de ensino e aprendizagem dos estudantes, por meio do uso de 
uma linguagem de programação visual [Fincher et al. 2010]. Entre as mais conhecidas são: Alice [Cooper, Dann e Pausch 2000], Greenfoot [Henriksen e Kölling 2004] e Scratch [Resnick et al. 2009]. Dessa forma, ambientes de ensino a programação estão sendo cada vez mais aceitos e adotados pelos educadores, como é o caso do Scratch, com intuito de minimizar esse problema. [Neto 2013] apresenta um estudo com a utilização do Scratch no inicio da disciplina de lógica, mostrando as atividades desenvolvidas por meio de um estudo de caso com alunos entre 16 e 17 anos em um curso de técnico em informática. $\mathrm{O}$ estudo mostrou que os alunos que apresentavam dificuldades de interpretar os problemas propostos em sala e criar soluções lógicas computacionais, acabaram despertando maior interesse após a inserção da ferramenta no ambiente de ensino.

Em [Mélo et al. 2011] os resultados mostram que os recursos visuais apresentados no Scratch facilitam a aprendizagem de lógica. Além disso, a motivação dos estudantes ao usar a ferramenta pode auxiliar em uma experiência de aprendizado melhor. Além disso, [Dias e Serrão 2014] revelam que o uso do Scratch pode auxiliar a diminuir os índices de evasão e reprovação nos cursos de informática.

Apesar de várias iniciativas de integração do Scratch, grande parte dos alunos relataram dificuldade de usar recursos da ferramenta, causada por elementos nãointuitivos [Melo et al. 2014; Neto 2013]. Dessa forma, baseado nos trabalhos citados, constatamos que muitas abordagens buscam analisar o Scratch como uma ferramenta para o ensino de programação [Aureliano e Tedesco 2012]. No entanto, não foi avaliado sobre o ponto de vista de sua interface. Dessa forma, buscamos, nesta pesquisa medir o grau de comunicabilidade da ferramenta do ponto de vista dos usuários com o objetivo de mensurar sua qualidade de interação.

\subsection{Caracterização do Aplicativo Scratch}

Em função da facilidade de uso e a interface agradável o Scratch tem se tornado um dos principais ambientes para o ensino de programação. Desenvolvido no Media Lab do Instituto de Tecnologia de Massachusetts, esse ambiente introdutório de ensino tem como objetivo facilitar a introdução de conceitos de matemática e computação, induzindo o pensamento criativo, o raciocínio sistemático e o trabalho colaborativo [About Scratch 2015]. Para isso, o software possui uma interface visual simples, conforme mostra a Figura1, levando de forma intuitiva, às principais estruturas de uma linguagem como: variáveis, operadores, estruturas de decisão e de repetição, e outros [Mélo 2011].

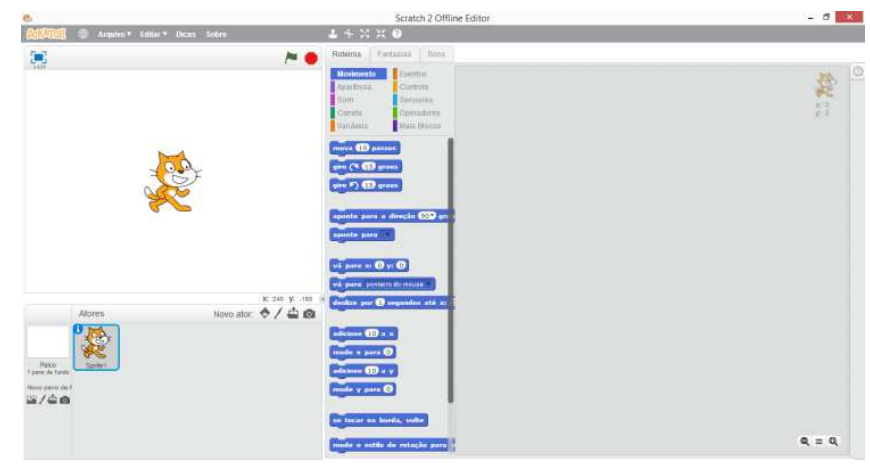

Figura 1. Tela principal do Scratch 
O Scratch utiliza a metodologia de clicar e arrastar por meio de blocos utilizando diversos tipos de mídias, possibilitando a criação de histórias interativas, animações, jogos, músicas e o compartilhamento dessas criações na Internet [Resnick, 2009]. Essas características tornam processo de iniciação é rápido e o usuário pode imediatamente conceber projetos, com maior ou menor grau de mediação de um professor.

Apesar de várias iniciativas de integração do Scratch, grande parte dos alunos relataram dificuldade de usar recursos da ferramenta, causada por elementos nãointuitivos [Melo et al. 2014; Neto, 2013]. Motivados por esse cenário, o artigo apresenta uma avaliação do impacto da usabilidade e comunicabilidade no ensino-aprendizagem de estudantes iniciantes. A descrição do estudo é descrita na próxima Seção.

\section{Avaliando a Qualidade de Uso do Scratch}

Para avaliar os princípios de comunicação e usabilidade da ferramenta Scratch foi adotado o método baseado na teoria da Engenharia Semiótica (EngSem) [Barbosa e Silva 2010] o Método de Avaliação da Comunicabilidade (MAC) [Prates et al. 2000], com o objetivo de analisar a metacomunicação transmitida do design para o usuário.

O MAC é um método que permite a observação de usuários em ambiente controlado. Assim, torna-se possível identificar os problemas de comunicabilidade (rupturas) a partir da recepção e entendimento da metamensagem do designer diretamente pelo usuário [De Souza, 2005]. Os usuários serão selecionados a partir de um perfil pré-definido, e executarão tarefas determinadas pelos avaliadores. Dessa forma, com a identificação e análise das rupturas de comunicação encontradas, pode-se sugerir melhorias que aumentem a qualidade da comunicação.

Essa característica centrada na utilização, com foco nos aspectos de interface, tornam o MAC uma importante abordagem de avaliação da interação. Além disso, é um método qualitativo e interpretativo que avalia a comunicabilidade a partir da recepção e entendimento da metamensagem pelo usuário. O propósito é avaliar se a interface da ferramenta alcançou os objetivos propostos para a qual foi desenvolvida.

O processo de avaliação do MAC possui uma sequência de passos para a realização da avaliação, dividido em três etapas: (1) Planejamento: que consiste na elaboração dos objetivos do teste, seleção dos participantes, criação das tarefas para teste, considerações sobre aspectos éticos, produção do material impresso para uso durante a avaliação, definição do cenário; (2) Coleção: coleta de dados é realizada através da execução do método e gravação das tarefas pelos participantes, anotações durante o teste e entrevista, através de um questionário, ao término do teste; (3) a Análise e Priorização: que é composta de três etapas [Prates et al. 2000]: 1) A etiquetagem são formas de interpretar a reação dos usuários às interrupções de comunicação durante o uso da ferramenta. As expressões são: Cadê?, O que é isto?, Epa!, Assim não dá, E agora?, Onde estou?, Por que não funciona?, Vai de outro jeito, Ué, o que houve?, Não, obrigado, Socorro!, Para mim está bom, Desisto; 2) Na fase de interpretação são identificadas e analisadas as falhas no processo de comunicação; 3) A geração do perfil semiótico é a fase de reconstrução da metamensagem do projetista ao usuário, onde serão apresentados os problemas encontrados em função das falhas identificadas. Para um melhor entendimento da aplicação do método de avaliação da comunicabilidade com a ferramenta Scratch dividiu-se a análise em três etapas distintas, apresentadas abaixo. 


\subsection{Fase I - Planejamento}

Para melhor compreensão, as etapas do processo de avaliação de comunicabilidade e usabilidade segue o paradigma descrito no formato GQM - Goal Question Metric [Basili e Rombach 1988], que foi analisar a ferramenta Scratch com o propósito de avaliar em relação à percepção sobre facilidade de uso, utilidade percebida e interface da aplicação, do ponto de vista dos alunos de Ciência da Computação, no contexto de utilizar a ferramenta como apoio a aprendizagem de lógica de programação.

Para o cenário de uso foi definido dois tipos de usuários diferentes: o Usuário Iniciante: estudantes de informática sem contato com programação; e Usuário Experiente: que são estudantes com contato com programação em disciplina. Dessa forma, foram selecionados 16 alunos, sendo oito alunos para cada tipo de classificação, o que representa um número adequado para que fosse realizado esse teste [Nielsen, 1993]. Os alunos aceitaram participar de forma voluntária

O perfil dos alunos classificados como Usuário Iniciante foi formado por alunos dos primeiros períodos de graduação do curso em Engenharia de Software, ingressantes no curso e ainda com o primeiro contato com as disciplinas de programação. Os alunos classificados como Usuário Experiente foram representados por alunos com contato com disciplinas de programação, a partir do quarto período, do mesmo curso. É importante ressaltar que os alunos não tinham contato anterior com o Scratch antes do teste. Isso porque o objetivo deste trabalho é analisar a comunicabilidade da ferramenta com diferentes níveis de conhecimento e perfis de usuário.

A documentação utilizada para registro da aplicação do MAC compreende: 1) Termo de Consentimento Livre Esclarecido: baseia-se no termo de aceitação permitindo a realização do teste e a divulgação dos dados estritamente para fins acadêmicos, sempre preservando a privacidade e o anonimato dos participantes; 2) Roteiro de Avaliação de Usabilidade: documento com o roteiro de tarefas para o usuário realizar para observação dos avaliadores durante a análise da ferramenta; 3) Questionário de Avaliação do Participante: relato descrito pelo participante sobre a experiência vivida com a avaliação do Scratch, e também, sua opinião sobre os aspectos de interface.

As atividades estratégicas realizadas para estudo no decorrer do teste foram descritas da seguinte forma: 1) Importar um ator da ferramenta; 2) Fazer troca de Sprite; 3) Fazer algum movimento; 4) Usar um sensor e um efeito sonoro; 5) Criar uma variável Score e; 6) Usar operadores. O teste foi projetado para ser realizado individualmente e assim ter uma avaliação mais detalhada das ocorrências. Foi utilizada a versão 2.0 do Scratch, que é a mais recente disponível para download ${ }^{1}$. Inicialmente foi feita uma explanação sobre a ferramenta para os alunos, dessa forma eles teriam um conhecimento prévio do que se tratava o programa. Os alunos foram orientados a indicar aos avaliadores o momento que iniciava uma respectiva tarefa e o momento em que ela terminava. Isso foi necessário para que os avaliadores pudessem acompanhar os passos que os alunos seguiam de acordo com o roteiro das tarefas, e assim, registrar com mais atenção as etiquetagens.

\footnotetext{
${ }^{1}$ http://scratch.mit.edu
} 


\subsection{Fase II - Execução}

A máquina utilizada para os testes foram dispostas no laboratório de informática do Instituto de Ciências Exatas e Tecnologia de Itacoatiara (ICET), e segue uma configuração básica e de baixo custo, uma vez que a ferramenta é multiplataforma e não requer requisitos avançados de hardware e software para ser utilizado.

Inicialmente foi apresentado aos alunos o Termo de Consentimento de Livre Esclarecido e somente depois de aceito e assinado o documento começava a avaliação. Para a realização dos testes foram dispostos dois avaliadores para registrar as ocorrências de etiquetagem e um para fazer a filmagem da avaliação. A filmagem era utilizada após o término do teste pelos avaliadores. Os vídeos foram úteis para transcrição e análise da interação de cada participante com o sistema. Dessa forma, era possível fazer uma análise mais eficiente das ocorrências percebidas nas etiquetagens.

Ao final de cada teste os alunos receberam o Questionário de Avaliação do Participante (QAP) e respondiam algumas questões relacionadas à experiência de uso do Scratch, (i.e. "A interface gráfica do Scratch lhe parece de fácil entendimento?"). O QAP também continha sugestões para propor melhorias na ferramenta, para uma avaliação qualitativa. Na coleta dos dados comparou-se os resultados da etiquetagem dos dois tipos de usuário: Usuário Iniciante e Usuário Experiente. Depois de analisados os registros de etiquetagem será avaliado o Questionário de Avaliação do participante e finalmente o cruzamento dos dados. Dessa forma, foi possível identificar o potencial da ferramenta quanto aos propósitos de interação e design.

\subsection{Fase III - Resultados}

Os resultados desta avaliação compreendem a análise realizada através da ferramenta de apoio a aprendizagem a programação para iniciantes, o Scratch. Portanto, a primeira avaliação foi feita com a aplicação do MAC para analisar a comunicabilidade e usabilidade da ferramenta. A Tabela 1 mostra o resultado da marcação das etiquetagens e a comparação entre os dois tipos de usuários.

Tabela 1. Registro das Etiquetagens do MAC

\begin{tabular}{|l|c|c|c|}
\hline \multicolumn{1}{|c|}{ Etiquetas } & Usuário Iniciante & Usuário Experiente & Total \\
\hline Cadê? & 17 & 15 & $\mathbf{3 2}$ \\
\hline E agora? & 14 & 10 & $\mathbf{2 4}$ \\
\hline Ué, o que houve? & 5 & 4 & $\mathbf{9}$ \\
\hline Epa! & 14 & 17 & $\mathbf{3 1}$ \\
\hline Assim não dá & 11 & 5 & $\mathbf{1 6}$ \\
\hline Onde estou? & 8 & 7 & $\mathbf{1 5}$ \\
\hline O que é isto? & 7 & 4 & $\mathbf{1 1}$ \\
\hline Por que não funciona? & 9 & 6 & $\mathbf{1 5}$ \\
\hline Socorro! & 9 & 3 & $\mathbf{1 2}$ \\
\hline Vai de outro jeito & 13 & 9 & $\mathbf{2 2}$ \\
\hline Não, obrigado & 6 & - & $\mathbf{6}$ \\
\hline Para mim está bom... & 3 & 2 & $\mathbf{5}$ \\
\hline Desisto & - & - & $\mathbf{1 9 8}$ \\
\hline Total & $\mathbf{1 1 6}$ & $\mathbf{8 2}$ & \\
\hline
\end{tabular}


A Tabela 1 mostra o resultado da soma dos registros das etiquetagens do MAC, em que a maior ocorrência foi da etiqueta identificada como Cadê?, no total de 32 rupturas. Foi observado que esse resultado ocorreu devido os usuários, tentarem inicialmente identificar a interface do aplicativo e reconhecer os símbolos dispostos na tela principal do programa. Outra etiqueta que obteve destaque foi a do Epa!, sendo a única marcação que os usuários experientes tiveram um número de ocorrências maior do que as do usuários iniciantes. O total de rupturas foi de 198 para todas as etiquetas.

O resultado obtido dessa segunda ocorrência ocorreu devido os usuários experientes demonstrarem ansiedade para realizar o teste e muitas vezes seguir por instinto uma ação. Dessa forma, os usuários reconheciam que era uma ação indesejada e retornavam imediatamente. Pode-se citar ainda um fato importante na última etiqueta, conhecida como Desisto, em que não foi registrada nenhuma ocorrência. Isso demonstra que os usuários não abandonaram a tarefa e tentaram concluir de alguma forma. O QAP foi aplicado pós-teste e analisado juntamente com os avaliadores do MAC. Dessa forma, foram elaboradas questões referentes ao teste e a experiência dos usuários ao utilizar a ferramenta, e assim, avaliar também quanto a sua percepção e facilidade de uso. Os dados do QAP foram analisados separadamente de acordo com os tipos de usuários classificados anteriormente e são apresentados na Tabela 2.

Tabela 2. Percepção dos usuários sobre o Scratch.

\begin{tabular}{|c|c|c|c|c|c|}
\hline \multirow{3}{*}{ Características } & \multirow{3}{*}{ Questões } & \multicolumn{4}{|c|}{$\begin{array}{c}\mathrm{N}^{\circ} \text { de Usuários } \\
(\%)\end{array}$} \\
\hline & & \multicolumn{2}{|c|}{ UI } & \multicolumn{2}{|c|}{$\mathbf{U E}$} \\
\hline & & $\begin{array}{c}S \\
(\%)\end{array}$ & $\begin{array}{l}\mathrm{N} \\
(\%)\end{array}$ & $\begin{array}{c}S \\
(\%)\end{array}$ & $\begin{array}{c}N \\
(\%)\end{array}$ \\
\hline \multirow{4}{*}{$\begin{array}{l}\text { Percepção de } \\
\text { aprendizado }\end{array}$} & $\begin{array}{l}\text { Você teve muita dificuldade em interpretar os } \\
\text { problemas propostos no teste? }\end{array}$ & 37,5 & 62,5 & 25 & 75 \\
\hline & $\begin{array}{l}\text { Você acredita que tem conhecimento lógico matemático } \\
\text { suficiente para trabalhar com a lógica de programação } \\
\text { do Scratch? }\end{array}$ & 75 & 25 & 100 & $\mathbf{0}$ \\
\hline & $\begin{array}{lccc}\text { Você } & \text { conseguiu imaginar soluções } & \text { lógicas } \\
\text { computacionais para os problemas propostos? } & \\
\end{array}$ & 100 & $\mathbf{0}$ & 100 & $\mathbf{0}$ \\
\hline & $\begin{array}{l}\text { A estrutura do software apresentou ferramentas com } \\
\text { termos e conceitos de lógicas difíceis de entender? }\end{array}$ & 25 & 75 & 12,5 & 87,5 \\
\hline \multirow{4}{*}{$\begin{array}{l}\text { Percepção de } \\
\text { facilidade de } \\
\text { uso }\end{array}$} & O software Scratch despertou seu interesse? & 75 & 25 & 100 & $\mathbf{0}$ \\
\hline & $\begin{array}{l}\text { A interface gráfica do Scratch lhe parece de fácil } \\
\text { entendimento? }\end{array}$ & 100 & $\mathbf{0}$ & 100 & $\mathbf{0}$ \\
\hline & $\begin{array}{l}\text { O software possui uma interface gráfica com algo que se } \\
\text { aproxima dos aplicativos usados por você no dia a dia? }\end{array}$ & 50 & 50 & 75 & 25 \\
\hline & O Scratch possui uma aparência interativa? & 75 & 25 & 100 & $\mathbf{0}$ \\
\hline \multirow{3}{*}{$\begin{array}{l}\text { Percepção de } \\
\text { utilidade do } \\
\text { Scratch }\end{array}$} & $\begin{array}{l}\text { O programa lhe causou um descontentamento e um } \\
\text { desanimo, ou em outras palavras, não lhe empolgou o } \\
\text { suficiente para um melhor aproveitamento? }\end{array}$ & 25 & 75 & $\mathbf{0}$ & 100 \\
\hline & Foi fácil realizar o teste com o Scratch? & 75 & 25 & 100 & $\mathbf{0}$ \\
\hline & $\begin{array}{l}\text { Você acredita que o Scratch vai lhe ajudar a ser capaz } \\
\text { de resolver problemas computacionais através do seu } \\
\text { uso? }\end{array}$ & 75 & 25 & 87,5 & 12,5 \\
\hline \multicolumn{6}{|l|}{$\begin{array}{l}\text { Legenda: } \\
\mathbf{S}=\operatorname{Sim} \\
\mathbf{N}=\text { Não }\end{array}$} \\
\hline
\end{tabular}

Pode-se notar na Tabela 2, que os usuários consideram que o aplicativo é útil para melhorar o seu aprendizado e que facilitaria a realização das atividades de 
programação. Entretanto, quatro usuários não consideram que o Scratch melhoraria a sua produtividade para realização das tarefas e aprendizado. Um usuário informou:

“Honestamente, não acho que isso irá melhorar no ensino específico". No entanto, é necessário realizar novos testes e comparar a utilização do Scratch com a formação de ensino comumente utilizada em sala de aula para verificar se o aplicativo pode auxiliar na melhoria do aprendizado dos alunos.

Outra característica analisada, na Tabela 2, foi em relação à Interface do Aplicativo, pode-se observar que houve discordâncias em todas as questões. Algumas afirmações dos usuários foram: "Não consegui usar o Scratch, pois não entendia nada"; "Alguns ícones estavam pequenos demais e não estavam visíveis na tela" $\mathrm{e}$ "Tive dificuldades apenas em relação aos botões que são dificeis de entender $e$ visualizar” e “(...) precisam de muito cliques para chegar em algumas funções”. Esses resultados indicam que melhorias na interface do Scratch podem melhorar a experiência no ensino, para aumentar a facilidade para reconhecer ou visualizar ícones, botões e nomenclaturas no aplicativo, diminuindo as dificuldades em utilizar o sistema.

Em relação a percepção de utilidade, a Tabela 2 mostra que o Scratch apresenta certa dificuldade e por isso não não possui índices de utilidade alto. um usuário sugeriu que "poderia adicionar tutorial de uso". As sugestões dadas permitiram a identificação de novas funcionalidades, o que possibilitará aos futuros usuários maior domínio sobre os assuntos apresentados em sala de aula. No entanto, observou-se que os usuários consideraram que após se acostumar com o Scratch, o mesmo apóia o ensino e aprendizagem, pois segundo um usuário "Na minha opinião este aplicativo pode ser muito útil para a aprendizagem de pessoas que estão a procura de um meio mais fácil de aprender a programar (...)”.

\section{Conclusões e Lições Aprendidas}

Este artigo apresentou um estudo de caso sobre uma experiência de uso do Scratch para ensino de programação, avaliando o impacto da usabilidade da ferramenta no aprendizado dos alunos iniciantes do curso de computação. A ferramenta tem como objetivo facilitar a introdução de conceitos de matemática e computação, induzindo o pensamento criativo, o raciocínio sistemático e colaboração [About Scratch 2015].

Por meio da avaliação quantitativa, observada através dos resultados das rupturas encontradas pelos usuários na avaliação do MAC visto na Tabela 1, tivemos um total de 198 ocorrências registradas sendo que, 116 dos usuários iniciantes e 82 dos usuários experientes. Segundo [Prates e Barbosa 2003] esses números mostram que de acordo com a relação dos tipos de falhas, apenas 5 ocorrências foram observadas como "Completas", sendo que a etiqueta "Desisto" não houve registro. O grupo de falhas ditas como "Parciais" obtiveram 28 ocorrências e as falhas classificadas como "Temporárias" registraram 165 ocorrências.

Dessa forma, os resultados mostram que a viabilidade do uso do Scratch é recomendada para qualquer tipo de usuário, mesmo para os que não têm muita experiência em programação. Outra contribuição importante é que apesar de haver uma diferença entre as afirmações, respondidas no questionário, não há uma diferença entre os modelos das amostras. No teste realizado encontramos algumas dificuldades, principalmente em relação à aceitação dos usuários voluntários para realizar os testes. 
Muitos alunos, mesmo os classificados como Usuários Experientes, ficaram com receio de realizar a avaliação pelo fato de pensar que não iriam concluir ou saber fazer as tarefas. Alguns declararam que mesmo cursando o segundo ano de graduação e já tendo certa experiência com disciplinas do curso de computação, não dominam muito bem a lógica de programação. Todas as dificuldades observadas nos permitiram demonstrar como a ferramenta Scratch é facilitador na vida dos alunos iniciantes nas disciplinas introdutórias de programação.

Para sugestões de melhorias os usuários relataram que apesar da ferramenta ter um tradutor, algumas áreas do programa não são alteradas como "Sprite", "Score". Dessa forma, quando o avaliador fez a leitura das tarefas com os usuários alguns tiveram dificuldade em encontrar a área destinada para realizar a ação. Esta dificuldade foi relatada pela maioria dos usuários iniciantes, que afirmam não ter um conhecimento amplo com a língua inglesa, o que dificultou a interpretação das tarefas.

Como trabalhos futuros pretende-se avaliar o potencial da ferramenta com alunos calouros do curso de computação, aplicando os conhecimentos sobre a ferramenta Scratch paralelamente a uma disciplina introdutória de programação. Esperase com isso, descrever os resultados do impacto causado pela ferramenta avaliando o desenvolvimento e o desempenho dos alunos durante os primeiros contatos com a lógica de programação através de uma linguagem de programação

\section{Referências}

Barbosa, S.D.J. and Silva, B.S. da., (2010). "Interação Humano-Computador". Elsevier Editora Ltda.

Basili, V., Rombach, H., (1988). "The tame project: towards improvement-oriented software environments". IEEE Transactions on Software Engineering, v. 14, n. 6, pp. 758 - 773.

Bonifácio, B. A.; Grana, C. B.; Coutinho, W.; Couto, E.; Fernandes, P. S. (2015) "Improving an education mobile application through usability evaluations", In: 9th International Technology, Education and Development Conference, Madrid, Espanha. v. 1. p. 30-42.

Boulic, R. and Renault, O. (1991) "3D Hierarchies for Animation", In: New Trends in Animation and Visualization, Edited by Nadia Magnenat-Thalmann and Daniel Thalmann, John Wiley \& Sons ltd., England.

Cooper, S.; Dann, W.; Pausch, R. (2000). "Alice: A 3-D tool for introductory programming concepts". Journal of Computing Sciences in Small Colleges, vol.15, n. 5, p.107-116.

Davis, F. "Perceived usefulness, perceived ease of use, and user acceptance of information technology". In MIS Quaterly, vol. 13, n.3. pp 319-339.

De Souza, C. S. (2005). "The Semiotic Engineering of Human-Computer Interaction". The MIT Press, Cambridge, MA.

Dias, K. L; Serrão, M. L. (2014). "A linguagem Scratch no ensino de programação: Um relato de experiência com alunos iniciantes do curso de licenciatura em computação". In: XXII WORKSHOP SOBRE EDUCAÇÃO EM COMPUTAÇÃO. Brasília.

Dyer, S., Martin, J. and Zulauf, J. (1995) "Motion Capture White Paper", http://reality.sgi.com/employees/jam_sb/mocap/MoCapWP_v2.0.html, December.

Fincher, S.; Cooper, S.; Kölling, M.; Maloney, J. (2010). "Comparing alice, greenfoot \& scratch”. Proceedings do 41st SIGCSE'10, Milwaukee, Wiscosin, USA. p. 192-193. 
Gomes, A.; Mendes, A. J. (2007) Learning to Program - Difficulties and Solutions. Proceedings do ICEE 2007, Coimbra, Portugal. Disponível em: <http://bit.ly/Lj3HLu>. Em: 05.05.2015

Henriksen, P.; Kölling, M. (2004). "Greenfoot: Combining object visualisation with interaction". Proceedings do 19thOOPSLA, Vancouver, Canadá. p. 73-82.

Holton, M. and Alexander, S. (1995) "Soft Cellular Modeling: A Technique for the Simulation of Non-rigid Materials", Computer Graphics: Developments in Virtual Environments, R. A. Earnshaw and J. A. Vince, England, Academic Press Ltd., p. 449-460.

Knuth, D. E. (1984), The TeXbook, Addison Wesley, $15^{\text {th }}$ edition.

Luna, J. Panach, J. Grigera, Rossi G. e O. Pastor, "Incorporating usability requirements in a test/model-driven web engineering approach", Journalof WebEngineering v. 9, n. 2, pp. 132156

Mélo, F. E N. (2011). "Do SCRATCH ao arduino: Uma proposta para o ensino introdutório de programação para cursos superiores de tecnologia". Congresso Brasileiro de Informática na Educação, Santa Catarina.

Nauman, M. and Uzair, M. (2007) "SE and CS Collaboration: Training Students for Engineering Large, Complex Systems". In: Conference on Software Engineering Education \& Training, Dublin. Proceedings. Washington: IEEE Computer Society, p. 167-174.

Neto, V. dos Santos M (2013). "A utilização da ferramenta SCRATCH como auxílio na aprendizagem de lógica de programação". Congresso Brasileiro de Informática na Educação, São Paulo.

Nielsen, J. (1993). Usability Engineering. Academic Press.

Pereira, P.de Sousa et. al (2012). "Análise do SCRATCH como ferramenta de auxílio ao ensino de programação de computadores". Congresso Brasileiro de Educação em Engenharia, Ceará.

Power T. and Shrestha P. (2010) "Exploration in the Context of Bangladesh", In: IADIS International Conference Mobile Learning, Porto, Portugal.

Prates, R. O., Souza, C. S. de, and Barbosa, S. D. J., (2000). "Methods and tools: a method for evaluating the communicability of user interfaces". Interactions 7, 31-38.

Resnick, M.; Maloney, J.; Monroy-Hernández, A.; Rusk, N.; Eastmond, E.; Brennan, K.; Millner, A.; Rosenbaum, E.; Silver, J.; Silverman, B.; Kafai, Y. (2009) "Scratch: programming for all". Communications of the ACM, vol. 52, n. 11, p. 60-67.

Rocha, P. S. et. al (2010). "Ensino e aprendizagem de programação: Análise da aplicação de proposta metodológica baseada no Sistema Personalizado de Ensino CINTED-UFRGS". Novas Tecnologias na Educação, Rio Grande do Sul.

Ronnie E.S.; Magalhães, Cleyton V. C.; Correia Neto, Jorge S.; Souza, Ellen P.R.; Vilar, Guilherme (2014) "Ferramentas, métodos e experiências no ensino de Engenharia de Software: um mapeamento sistemático", In: XXV Simpósio Brasileiro de Informática na Educação.

Scratch. About Scratch (Scratch Documentation Site) (2015). http://info.scratch.mit.edu/ About_Scratch Acesso em: 10.05.2015

Smith, A. and Jones, B. (1999). "On the complexity of computing". In Advances in Computer Science, pages 555-566. Publishing Press.

Wang, M., et al. 2009. The impact of mobile learning on students' learning behaviours and performance: Report from large blended classroom. Journal of Educational Technology, Vol. 1 N. 1, p. 673-695 